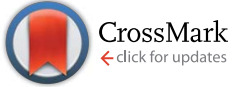

Cite this: Sustainable Energy Fuels, 2017, 1, 336

Received 11th November 2016 Accepted 12th December 2016

DOI: $10.1039 / c 6 s e 00050 a$

rsc.li/sustainable-energy

\section{Enhanced photoelectrochemical water splitting using oxidized mass-selected Ti nanoclusters on metal oxide photoelectrodes $\uparrow$}

\author{
Andrew Mclnnes, ${ }^{\text {ab }}$ Simon R. Plant, ${ }^{\text {b }}$ Isabel Mecking Ornelas, ${ }^{b}$ Richard E. Palmer ${ }^{b}$ \\ and K. G. Upul Wijayantha*a
}

\begin{abstract}
We report an enhancement of up to $85 \%$ in the photocurrent generated from a bismuth vanadate photoanode through the prior deposition of mass-selected Ti nanoclusters onto the semiconductor surface. We studied the effect of a variety of cluster sizes, deposited at the same density and with the same energy (1.5 keV per cluster), over the surface of separate $\mathrm{BiVO}_{4}$ photoanodes in a cluster beam source. Using mass-selected clusters of a narrow size distribution, we were able to reveal that the photocurrent is strongly dependent on the cluster size (in the size regime examined), leading to an increase of up to $85 \%$ in the photocurrent for $\mathrm{Ti}_{2000 \pm 54}$ clusters. Remarkably the quantities of metal used to achieve such an enhancement are on the $2.8 \times 10^{-7} \mathrm{~g} \mathrm{~cm}^{-2}$ level, resulting from the optimum density which is approximately 0.4 monolayers. This work highlights the importance of submonolayer surface treatments, using accurate mass-selected nanoclusters, for the modification of semiconductor surfaces in order to improve the interfacial charge transfer properties.
\end{abstract}

\section{Introduction}

The recent resurgence of interest in photoelectrolysis to generate solar fuels is underpinned by the pressing need to develop zero-carbon routes for production of chemical feedstocks. ${ }^{1} \mathrm{TiO}_{2}$ has been a material of significant interest for photoelectrolysis since the concept was first demonstrated in 1972. ${ }^{2}$ The anatase form of $\mathrm{TiO}_{2}$ has excellent electrical and optical properties, whilst also being one of the most chemically stable and abundant materials for solar water splitting. ${ }^{3-6}$ Since then, extensive studies have been conducted to investigate light harvesting and catalytic properties of $\mathrm{TiO}_{2}{ }^{7-9}$ Nanoscale $\mathrm{TiO}_{2}$ has also been shown to provide enhanced surface adsorption of reactant molecules, improved selectivity and a greater internal surface area. ${ }^{10}$ Size and texture controlled $\mathrm{TiO}_{2}$ (i.e. nanotube arrays, nanorods, quantum dots) has been widely used for this purpose and enhanced photoelectrochemical properties have been reported. ${ }^{11,12}$ Mass-selection, in the $1-10 \mathrm{~nm}$ size range in particular, offers greater control over particle size, surface and

${ }^{a}$ Energy Research Laboratory, Department of Chemistry, Loughborough University, Loughborough, LE11 3TU, UK. E-mail: u.wijayantha@lboro.ac.uk

${ }^{b}$ Nanoscale Physics Research Laboratory, School of Physics and Astronomy, University of Birmingham, Edgbaston, B15 2TT, UK

$\dagger$ Electronic supplementary information (ESI) available: Mass spectrum showing no target oxidation; examples of processed STEM images for $\mathrm{Ti}_{2000}$ and $\mathrm{Ti}_{8000}$; XPS survey for cluster modified $\mathrm{BiVO}_{4} ; J-V$ curve for $\mathrm{Ti}_{923}$ modified $\mathrm{BiVO}_{4} ; J-V$ for $\mathrm{Ti}_{8000}$ modified $\mathrm{BiVO}_{4}$; stability $J V$ curves for $\mathrm{BiVO}_{4}$ under white light and blue light illumination; $J-V$ curve for cluster modified FTO control experiment. See DOI: $10.1039 / \mathrm{c} 6 \mathrm{se} 00050 \mathrm{a}$ texture. Mass-selection may also offer the ability to optimize the electronic, specific surface and selective properties, all of which can dramatically vary with cluster size, and which may enhance the selectivity and rate of certain chemical reactions. ${ }^{13,14}$ For example, recently Perez-Alonso utilized mass-selected Pt clusters to study the effect of particle size on the activity of Pt for the oxygen reduction reaction. ${ }^{15}$ They found a strong correlation between size and activity, which was linked to the number of terrace sites present on the Pt cluster. The work was in close agreement with theoretical calculations and allowed fabrication of optimally sized particles rich in desired terrace sites. Recently, we have shown that photoelectrolysis can be influenced by controlling the surface termination of the semiconductor $\mathrm{Fe}_{2} \mathrm{O}_{3} .{ }^{16}$

Notably very few investigations have been reported for the application of mass-selected $\mathrm{TiO}_{2}$ nanoparticles in photoelectrolysis. ${ }^{17-20}$ Mass control of nanoclusters can be achieved through careful optimization of the production method. A wide variety of physical and chemical methods have been studied for production of such nanoclusters. ${ }^{21-26}$ In particular, physical methods that produce ion beams, such as laser ablation and magnetron sputtering, can also utilize a mass filter, such as a quadrupole ${ }^{27}$ or time of flight (TOF) mass filters, ${ }^{28,29}$ to achieve fine resolution of cluster mass. In addition, mass-selected ion beams also allow the control of coverage at monolayer, submonolayer or multilayer regimes with high accuracy by altering parameters such as particle mass, deposition energy and average densities. In a recent work, mass-selected $\mathrm{TiO}_{2}$ clusters produced using a quadrupole mass filter were deposited on 
H-terminated Si substrates and photoelectrochemical properties of these electrodes were studied under illumination from a xenon lamp in $5 \mathrm{M} \mathrm{KOH} .^{30}$ The authors found enhanced photocurrent with decreasing cluster size, whereby use of $4 \mathrm{~nm}$ clusters showed nearly twice the photocurrent than $8 \mathrm{~nm}$ clusters. The authors attribute this photocurrent increase to greater surface area presented by the smaller clusters and an increase in amorphicity. With the exception of this, mass-selected clusters have been largely unused in photoelectrolysis, primarily due to the difficulty in controlling cluster mass accurately at the preparation stage. In fact, to the best of our knowledge, there have been no studies on the deposition of mass-selected $\mathrm{Ti}$ nanoclusters on metal oxide semiconductor electrode surfaces for the study of their photoelectrochemical properties.

Herein, we report the photoelectrochemical study of oxidized mass-selected Ti nanoclusters deposited on bismuth vanadate, $\mathrm{BiVO}_{4}$, photoelectrodes. $\mathrm{BiVO}_{4}$ has received significant interest recently and is considered as one of the most promising anodic semiconductor materials for photoelectrolysis, hence it was chosen for this investigation. ${ }^{31-33}$ Some of the best literature reported $\mathrm{BiVO}_{4}$ photoelectrodes have been achieved utilizing heterojunctions, particularly those of $\mathrm{BiVO}_{4}$ and $\mathrm{WO}_{3}$, giving photocurrent up to 5.35 and $6.72 \mathrm{~mA} \mathrm{~cm}^{-2}$ in two independent works, respectively. ${ }^{34,35}$ Additionally texturing (to increase the surface area) and tungsten doping of $\mathrm{BiVO}_{4}$ have also been extremely popular techniques to enhance photocurrent. ${ }^{36}$ Plain $\mathrm{BiVO}_{4}$ produced by AACVD has a lower photocurrent than this, but still reasonable performance compared to other plain $\mathrm{BiVO}_{4}$ in the literature. ${ }^{32,37}$ AACVD is a scalable technique that has been used previously to make highly nanostructured metal oxide photoelectrodes. ${ }^{38,39}$ In this study a significant enhancement of photocurrent density of $\mathrm{BiVO}_{4}$ photoelectrodes has been observed through the controlled deposition and subsequent oxidation of mass-selected $(\mathrm{Ti})_{N}(N=923,2000$ or 8000) clusters. It was found that the photocurrent enhancement by utilizing clusters was mass dependent, with an increase of $85 \%$ by the oxidized $\mathrm{Ti}_{2000 \pm 54}$ clusters compared with bare $\mathrm{BiVO}_{4}$.

\section{Methods}

Bismuth vanadate photoelectrodes were prepared by aerosol assisted chemical vapor deposition (AACVD) on fluorine-doped tin oxide conducting glass substrates, as reported previously. ${ }^{32}$ Briefly, bismuth nitrate pentahydrate $(7.5 \mathrm{mmol})$ was dissolved in the minimum amount of acetyl acetonate. This was added to a solution of vanadyl acetyl acetonate $(7.5 \mathrm{mmol})$ in methanol, with further methanol added to achieve a volume of $250 \mathrm{~cm}^{3}$ and a final concentration of $0.03 \mathrm{~mol} \mathrm{dm}^{-3}$. The solution was gently heated at $70{ }^{\circ} \mathrm{C}$ for one hour whilst stirring. Commercially available FTO glass (TEC 8 Pilkington, $8 \Omega$ sq. $^{-1}$ ) was used as the substrate. Substrate slides $(1 \times 2 \mathrm{~cm})$ were cut manually with a diamond tipped cutter and ultrasonically cleaned in 18 $\mathrm{M} \Omega$ distilled water, acetone, propan-2-ol and then stored in ethanol. Before deposition by AACVD, the substrates were removed from the ethanol, dried and then placed in the AACVD deposition chamber. Samples were heated to $500{ }^{\circ} \mathrm{C}$ for 15 minutes in air before deposition. Typically $20 \mathrm{~cm}^{3}$ of precursor was placed in a round-bottom flask above the piezoelectric modulator of an ultrasonic humidifier (PIFCO ultrasonic humidifier) to generate the aerosol. Air was used as the carrier gas (compressed, BOC gases) at a flow rate of $175 \mathrm{~cm}^{3} \mathrm{~min}^{-1}$ (Air flowmeter, GPE scientific limited) to transfer the aerosol into the second chamber, where it was then mixed with a second flow of air at $2340 \mathrm{~cm}^{3} \mathrm{~min}^{-1}$. The aerosol droplets are then decomposed in the AACVD chamber to produce a thin film on the FTO substrate. The process was continued for 2 hours to produce a film with a uniform thickness.

Mass-selected Ti clusters were produced using a magnetronsputtering and gas-aggregation cluster beam source ${ }^{25}$ incorporating a lateral time-of-flight (TOF) mass selector, ${ }^{29}$ as shown in Fig. 1. The clusters were generated through the sputtering of a Ti target (99.99\% Ti, Pi-Kem Ltd.) in a UHV-compatible system, cooled with liquid nitrogen. The cluster source is comprised of 3 main chambers for cluster generation, cluster ion extraction and beam formation and mass selection, respectively. These are followed by a smaller chamber used for sample deposition. FTO glass samples were each clamped to a steel sample plate which is slotted into the deposition stage within the chamber. The focused, mass-selected cluster beam impinges normal to the surface of the substrate. The sample arm is connected to a motorized platform which allows automated rastering of the sample in front of the beam. The general approach followed was to optimize the formation parameters iteratively to achieve the highest beam currents possible. Typical parameters including DC magnetron power $(150 \mathrm{~W})$, Ar flow rate $(180 \mathrm{sccm})$, He flow rate $(200 \mathrm{sccm})$ and condensation length $(250 \mathrm{~mm})$ were used to achieve beam currents of approximately $100 \mathrm{pA}$. The magnetron settings were kept constant but the chamber pressure was modified, between 0.74-4.5 mbar, to improve the yield of smaller and larger cluster sizes respectively.

The $\mathrm{Ti}_{N}{ }^{+}$clusters were mass-selected prior to deposition onto the FTO substrates (high vacuum conditions, $10^{-7}$ to $10^{-6} \mathrm{mbar}$ ), which were biased so as to achieve a landing energy of $1500 \mathrm{eV}$ per cluster. Each cluster size was deposited on a separate FTO substrate. The TOF mass-filter was set to provide a mass resolution of $\pm 2.7 \%$, as determined through calibration using a beam of $\mathrm{Ar}^{+}$ions. This resolution yields mass-selected clusters of $\mathrm{Ti}_{923 \pm 25}, \mathrm{Ti}_{2000 \pm 54}$, and $\mathrm{Ti}_{8000 \pm 216}$ for clusters with nominally 923 , 2000 and $8000 \mathrm{Ti}$ atoms. These labels, $\mathrm{Ti}_{923 \pm 25}, \mathrm{Ti}_{2000 \pm 54}$, and $\mathrm{Ti}_{8000 \pm 216}$, will be used from this point onwards to discuss the respective cluster sizes. In order to ensure a uniform distribution of clusters over the entire surface of the photoelectrode, the

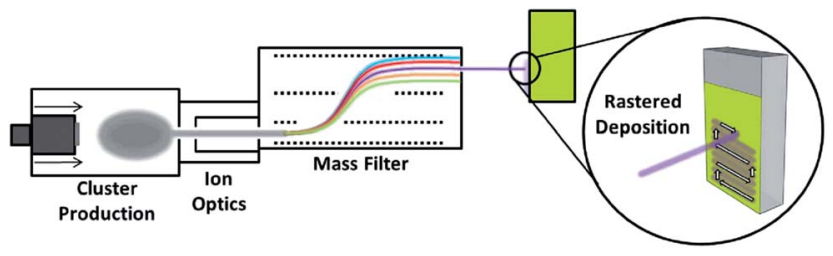

Fig. 1 Schematic diagram of the cluster beam source used for depositing an even coverage of mass-selected Ti nanoclusters on $\mathrm{BiVO}_{4}$ photoelectrodes. 
bismuth vanadate films $\left(5 \times 10 \mathrm{~mm}^{2}\right)$ were rastered with respect to the cluster beam. Here, the process of rastering involves moving the sample in front of the beam in an automated fashion, depositing mass-selected clusters onto the substrate line-by-line whilst the beam is maintained at a fixed position.

The beam current of mass-selected clusters is monitored at the substrate using a picoammeter during deposition, and hence, the average density is determined by calculating the total dose (beam current integrating with respect to time) over the deposition area of the substrate. Typically beam currents approaching $100 \mathrm{pA}$ could be achieved, leading to deposition times of 42 minutes for an average density of $3.5 \times 10^{10}$ clusters per $\mathrm{mm}^{2}$. Following the deposition, substrates were brought into air and allowed to oxidize.

The current density-voltage $(J-V)$ characteristics of the $\mathrm{BiVO}_{4}$ thin films, with and without surface attachment of clusters, were tested using a Galvanostat/Potentiostat (Eco Chemie micro-Autolab type III), under illumination with an AM 1.5 Class A solar simulator (Solar Light $16 \mathrm{~S}$ - 300 solar simulator), at $100 \mathrm{~mW} \mathrm{~cm}^{2}$ light intensity, calibrated by a silicon pyranometer (Solar Light Co., PMA2144 Class II). The thin films were measured in a standard three-electrode configuration in a quartz cell using a platinum wire counter-electrode and a Ag| $\mathrm{AgCl} / 3 \mathrm{M} \mathrm{KCl}$ reference electrode. The electrolyte was $1 \mathrm{M}$ sodium sulfate and the scan rate was maintained at $10 \mathrm{mV} \mathrm{s}^{-1}$ to record the $J-V$ plots. PEIS measurements were conducted using a collimated blue light LED $(\lambda=455 \mathrm{~nm}$, ThorLabs Ltd. $\mathrm{UK}$ ), at an applied potential of $1.23 \mathrm{~V} v s$. RHE, over a $100 \mathrm{mHz}$ to $10 \mathrm{kHz}$ frequency range for $\mathrm{BiVO}_{4}$ electrodes with and without cluster deposition. The measured data were fitted using an equivalent circuit utilizing a resistor in series with a resistor and constant phase element in parallel on the Nova software (Metrohm Autolab B.V.). STEM analysis was carried out using a 200 $\mathrm{kV}$ JEOL 2100F STEM with a spherical aberration corrector (CEOS) using a High Angle Annular Dark Field Detector with inner and outer collection angles of 62 and $164 \mathrm{mrad}$ respectively. Samples were deposited on graphene oxide coated lacey carbon on 300 mesh copper TEM grids (EM Resolutions Ltd.) and image acquisition times were approximately 10 s. XPS spectra were recorded using a Thermo Scientific K-Alpha XPS spectrometer operated with an unmonochromated Al K $\alpha$ X-ray source $(1486.6 \mathrm{eV})$. The surface morphology of $\mathrm{BiVO}_{4}$ thin films was studied using a Leo $1530 \mathrm{VP}$ field emission gun scanning electron microscope (FEG-SEM) at an accelerating voltage of $5 \mathrm{kV}$ and working distance of $5 \mathrm{~mm}$.

\section{Results and discussion}

Mass-selected Ti nanoclusters were prepared by a DC-sputtering gas-aggregation cluster beam source and a schematic of the cluster source is shown in Fig. 1. Mass analysis of Ti nanoclusters (prepared by the DC-sputtering gas-aggregation cluster beam source) was conducted using an in-built mass spectrometer. Relatively small mass-controlled Ti nanoclusters (in the range of 2-8 atoms per cluster) can be readily produced by utilizing the pressure $\sim 0.54$ mbar in the generation chamber, whilst keeping all other parameters constant, as shown in Fig. 2A.
By increasing the pressure in the generation chamber up to 4.5 mbar, the atomic mass distribution of nanoclusters can be increased. The longest aggregation distance available in the cluster source was $250 \mathrm{~mm}$ and it was used in order to facilitate production of larger clusters. The shift in the peak in beam current, as shown in Fig. 2B, shows that the key parameters, such as pressure, ion optics and aggregation distance, can be tuned to favor the production of clusters with a larger mass. Thus, by controlling these parameters, mass-selected Ti clusters can be tuned for, and produced, with up to $10000 \mathrm{Ti}$ atoms per cluster. The diameter of individual titanium clusters has been estimated using a spherical cluster approximation and the bulk density of Ti, leading to values of $3.14,4.07$, and $6.5 \mathrm{~nm}$ for $\mathrm{Ti}_{923 \pm 25}, \mathrm{Ti}_{2000 \pm 54}$, and $\mathrm{Ti}_{8000 \pm 216}$ clusters, respectively. ${ }^{40}$

Mass-selected Ti clusters were deposited on graphene oxide coated TEM grids to allow visualization of the clusters by highangle annular dark field (HAADF) scanning transition electron microscopy (STEM). ${ }^{\mathbf{4 1 - 4 3}}$ Samples were not protected prior to imaging, i.e. the conditions are similar to those used for cluster deposition on $\mathrm{BiVO}_{4}$ photoelectrodes. It is therefore expected that Ti nanoclusters will have undergone full oxidation and be converted to $\mathrm{TiO}_{2}$ clusters. In fact, this has been experimentally confirmed by XPS analysis.
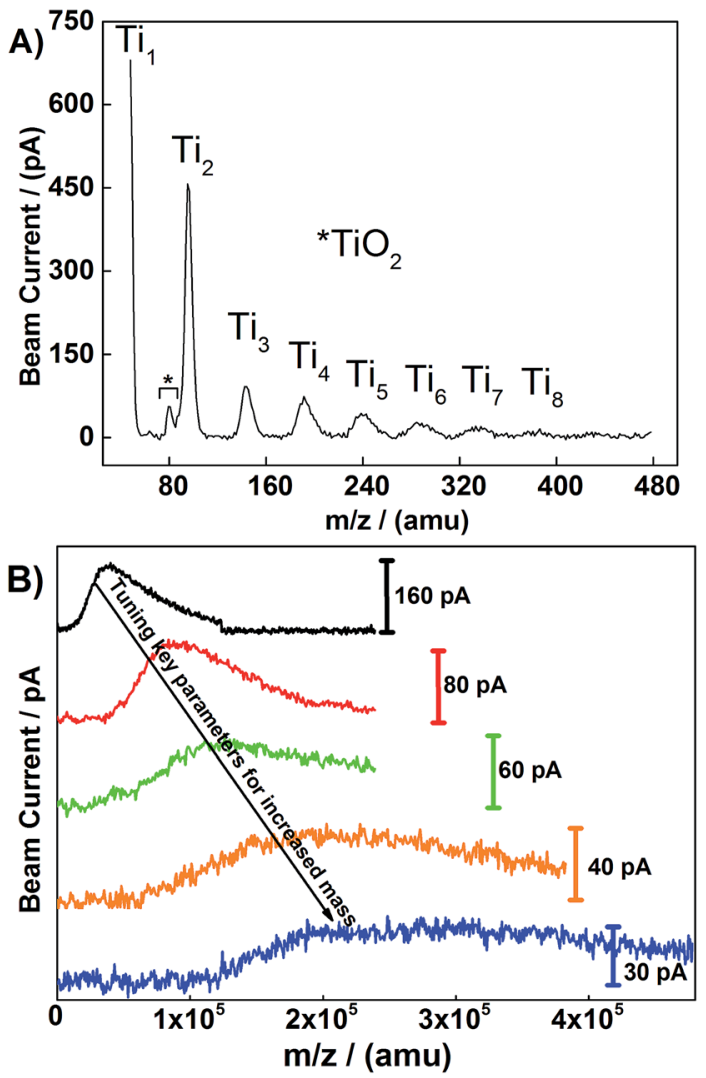

Fig. 2 (A) A mass spectrum over a low mass range showing the presence of $\mathrm{Ti}_{1}-\mathrm{Ti}_{8}$. The peak at approx. $80 \mathrm{amu}$ is attributed to surface oxide on the target, which is removed after a period of sputtering; (B) mass spectra showing the optimization of cluster beam production with tuning parameters set for cluster sizes of $\mathrm{Ti}_{700}$ (black), $\mathrm{Ti}_{2000}$ (red), $\mathrm{Ti}_{4000}$ (green), $\mathrm{Ti}_{5000}$ (orange) and $\mathrm{Ti}_{10} 000$ (blue). 
From close inspection of Fig. 3A-C, one can discern the difference in shape of the clusters with the increasing cluster size. Small clusters, such as $\mathrm{Ti}_{923 \pm 25}$, appear to be quasispherical whilst larger clusters, such as $\mathrm{Ti}_{8000 \pm 216}$, are far more irregular in shape. This change in shape can be attributed to the cluster formation mechanism in the condensation chamber, where the larger clusters are formed by merging several smaller clusters. ${ }^{44}$ High magnification STEM images of the oxidized clusters (given in inserts of Fig. 3A-C) provide evidence that the deposited clusters are amorphous.

Analysis of the HAADF intensity can be used to determine the size distribution of clusters present on the TEM grids, since the intensity distributions are proportional to the nuclearity for a given stoichiometry. ${ }^{\mathbf{4 1 , 4 4}}$ From Fig. $3 \mathrm{D}$ it is clear that nominal $\mathrm{Ti}_{923 \pm 25}$ and $\mathrm{Ti}_{2000 \pm 54}$ samples have more narrow size distributions, whilst $\mathrm{Ti}_{8000 \pm 216}$ has a much broader distribution. The Full-Width Half-Maximum (FWHM) values for the nominal $\mathrm{Ti}_{923 \pm 25}, \mathrm{Ti}_{2000 \pm 54}$ and $\mathrm{Ti}_{8000 \pm 216}$ clusters are in all cases very much larger than the nominal size ranges and are equivalent to 456.7, 504.2 and 1196.0 Ti atoms respectively. The likely main reasons for the differences in the widths are (i) the additional broadening error associated with the STEM intensity measurements including background subtraction and (ii) some tendency for aggregation or limited sintering of the clusters on the surface in high coverage areas. The peak at 4000 atoms for the $\mathrm{Ti}_{2000 \pm 54}$ clusters may be a combination of binaries created by coalescence and double mass/double charge clusters transmitted by the mass filter. ${ }^{45}$

XPS analysis was carried out on bismuth vanadate photoelectrodes coated with the mass-selected $\mathrm{Ti}_{923 \pm 25}$ and $\mathrm{Ti}_{2000 \pm 54}$ clusters to determine the elemental composition on the surface before and after the photoelectrochemical measurements

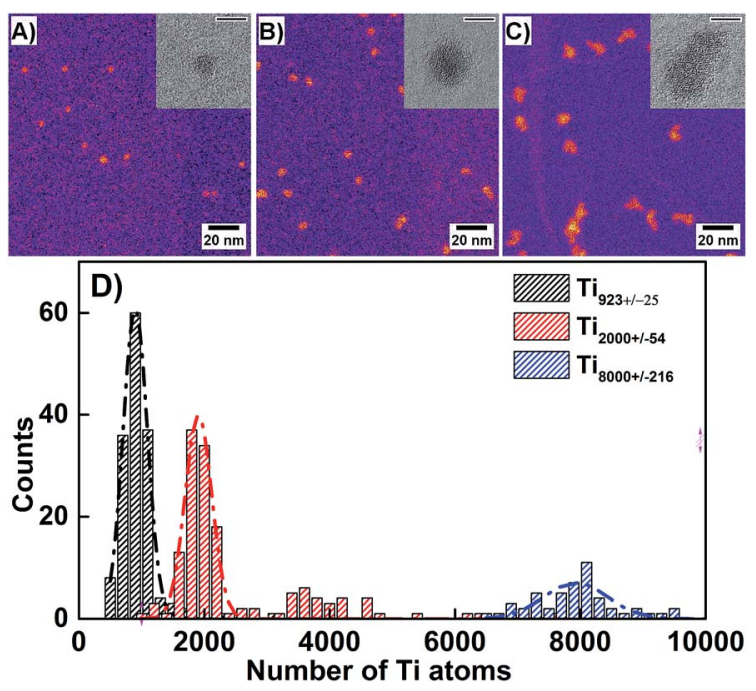

Fig. 3 (A-C) HAADF STEM images of oxidized nominal $\mathrm{Ti}_{923 \pm 25}$, $\mathrm{Ti}_{2000 \pm 54}$ and $\mathrm{Ti}_{8000 \pm 216}$ clusters, respectively (1M× magnification). Corresponding high magnification bright field STEM images are given in inset of each image with $5 \mathrm{~nm}$ scale bar. (D) Intensity distribution of as-deposited mass-selected $\mathrm{Ti}$ clusters calculated from HAADF intensity data normalized to the number of $\mathrm{Ti}$ atoms present in the cluster.
(Fig. 4). A prominent peak for Ti $2 \mathrm{p}_{3 / 2}$ is observed at $458.48 \mathrm{eV}$, in Fig. 4A, for $\mathrm{Ti}_{2000 \pm 54}$ clusters deposited on $\mathrm{BiVO}_{4}$. Previous reports indicate that Ti $2 \mathrm{p}_{3 / 2}$ peaks at $458.8 \mathrm{eV}$ represent $\mathrm{Ti}^{4+}$ in $\mathrm{TiO}_{2}$, around $457 \mathrm{eV}$ for TiO and $454 \mathrm{eV}$ for Ti metal. ${ }^{46,47}$ Thus the peak at $458.48 \mathrm{eV}$ suggests clusters have fully oxidized to form $\mathrm{TiO}_{2}$. The surface of $\mathrm{Ti}$ is known to readily oxidize and form its native oxide and our XPS data provide evidence to suggest that, in atmospheric conditions, clusters of the size range used in this study undergo complete oxidation shortly after being removed from the high vacuum chamber. ${ }^{42}$ The peak present at $464 \mathrm{eV}$ in Fig. 4A corresponds to an overlap between Ti $2 \mathrm{p}_{1}$ and $\mathrm{Bi} 4 \mathrm{~d}_{3}$. Prominent peaks at 529.88 and $531.88 \mathrm{eV}$ correspond to oxygen in the $\mathrm{BiVO}_{4}$ and $\mathrm{TiO}_{2}$ respectively in Fig. $4 \mathrm{~B} .{ }^{48} \mathrm{Ti}$ peaks can still be seen on samples that have undergone photoelectrochemical measurements in liquid electrolytes, as shown in Fig. 4C. This suggests that clusters are well adhered to the surface of $\mathrm{BiVO}_{4}$. For samples deposited with smaller clusters, such as $\mathrm{Ti}_{923 \pm 25}$, Ti peaks are seen in the same energy range, although at a lower intensity (Fig. 4D). Imaging clusters by microscopic methods on the surface of $\mathrm{BiVO}_{4}$ photoelectrodes was difficult due to their smaller size, however XPS provides sufficient evidence for the presence of clusters on the surface.

The photoelectrochemical properties of bismuth vanadate photoelectrodes modified with mass-selected oxidized Ti clusters were studied by recording steady-state current density-voltage plots under AM 1.5 simulated light. Electrodes were illuminated from the substrate side. The illumination was interrupted periodically, known as chopping, in order to observe the light and dark current simultaneously. A typical "chopped" $J-V$ curve recorded for a $\mathrm{BiVO}_{4}$ electrode modified with $\mathrm{Ti}_{2000 \pm 54}$ clusters is shown in Fig. 5B. Provided there are no other redox reactions occurring, the photocurrent is representative of the degree of oxygen evolution reaction (OER) occurring at the $\mathrm{BiVO}_{4}$-electrolyte interface. Deposition of mass-selected nanoclusters has resulted in an increase of the photocurrent density of $\mathrm{BiVO}_{4}$ over almost the entire potential window investigated. The percentage photocurrent increase is not uniform throughout the potential window, with a greater percentage increase observed at relatively low applied voltages for the modified $\mathrm{BiVO}_{4}$ electrode. The absence of extra redox features in the dark after incorporation of the clusters on the $\mathrm{BiVO}_{4}$ electrode surface indicates that only the light induced-charge transfer at the interface is influenced by the addition of clusters. Interestingly, the enhancement of photocurrent is observed for all sizes of mass-selected clusters used in our study. However, a characteristic negative shift of the photocurrent onset, corresponding to a catalytic effect, was not seen. ${ }^{\mathbf{4 9}}$ This indicates that the role of clusters may not be catalytic in nature. But the enhancement of the photocurrent suggests the clusters could be influencing charge transfer kinetics favorably at the electrode/electrolyte interface. It is well known that the kinetics of the OER are slow, as 4 electrons are required to produce 1 molecule of $\mathrm{O}_{2}$. In fact, the kinetic bottleneck of OER is one of the key limiting factors in the overall photoelectrolysis. ${ }^{50}$

Our observations also indicate that the photocurrent enhancement is dependent on the size of the mass-selected clusters used to modify the $\mathrm{BiVO}_{4}$ surface (Fig. 5D). As evident in 

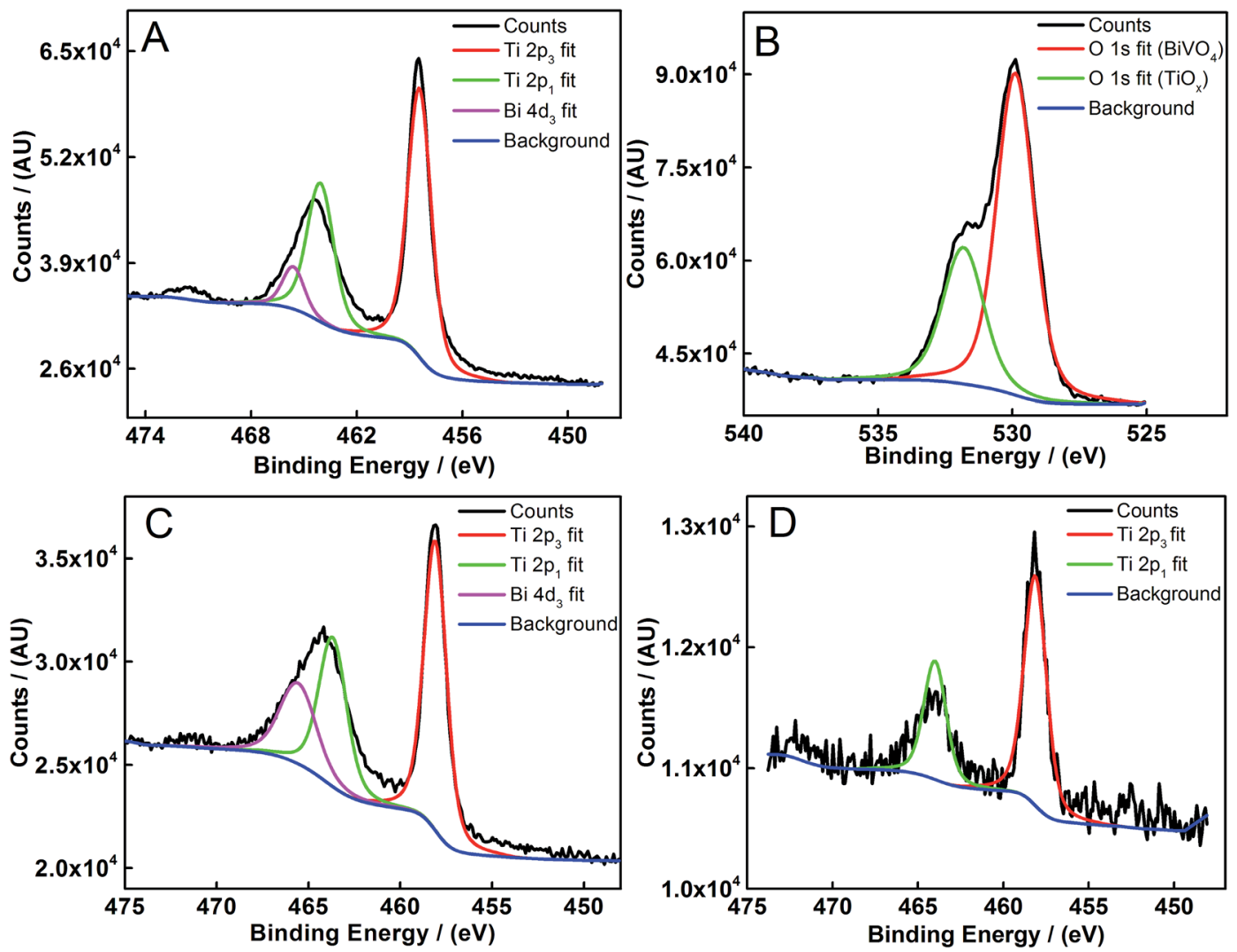

Fig. 4 XPS spectra of (A) $\mathrm{Ti}_{2000 \pm 54}$ deposited on $\mathrm{BiVO}_{4}$ with a peak corresponding to $\mathrm{Ti}_{2} 2 \mathrm{p}_{3}\left(458 \mathrm{eV}\right.$ ); (B) the same BiVO ${ }_{4}$ electrode exhibiting oxygen peaks at $529 \mathrm{eV}$ and $531 \mathrm{eV}$ corresponding to $\mathrm{BiVO}_{4}$ and $\mathrm{TiO}_{2}$, respectively; (C) a BiVO 4 photoelectrode with Ti $2000 \pm 54$ clusters after photoelectrochemical measurements; (D) a $\mathrm{BiVO}_{4}$ photoelectrode with $\mathrm{Ti}_{923 \pm 25}$ clusters deposited on the surface after photoelectrochemical measurements. All cluster samples were deposited at an average density of $3.5 \times 10^{10}$ clusters per $\mathrm{mm}^{2}$.

Fig. 5B and $\mathrm{D}$, the highest photocurrent increase is obtained when the $\mathrm{BiVO}_{4}$ electrode surface is modified with $\mathrm{Ti}_{2000 \pm 54}$ clusters. This accounts for an $85 \%$ photocurrent improvement at $1.23 \mathrm{~V} v$ s. RHE. As shown in Fig. $5 \mathrm{~A}$, there is no significant change in optical absorbance of $\mathrm{BiVO}_{4}$ electrodes before and after cluster incorporation and is therefore not due to an increase in visible light absorption due to clusters. Thus the significant photocurrent improvement could be due to the increased photoinduced charge transfer rate at the cluster incorporated $\mathrm{BiVO}_{4} /$ electrolyte interface compared to that of a bare $\mathrm{BiVO}_{4} /$ electrolyte interface.

In order to further investigate the increased photocurrent density after incorporation of clusters on the $\mathrm{BiVO}_{4}$ electrode surface, the effect of photocurrent enhancement as a function of average density of clusters was studied. The average density of $\mathrm{Ti}_{2000 \pm 54}$ clusters was varied from between 0.2 and $7.5 \times 10^{10}$ clusters per $\mathrm{mm}^{2}$ by preparing a series of identical $\mathrm{BiVO}_{4}$ electrodes containing appropriate cluster densities. In order to evaluate the photocurrent enhancement over the entire voltage window used, two representative voltages were selected $(1.23 \mathrm{~V}$ and $1.9 \mathrm{~V} v s$. RHE). As shown in Fig. 6, the percentage photocurrent increase resulting from $\mathrm{Ti}_{2000 \pm 54}$ cluster deposition on $\mathrm{BiVO}_{4}$ as a function of average density was analyzed at both $1.23 \mathrm{~V}$ and $1.9 \mathrm{~V} v$ s. RHE. The highest increase of photocurrent density for $1.23 \mathrm{~V}$ and $1.9 \mathrm{~V}$ vs. RHE was observed when the average density of clusters is adjusted to $3.5 \times 10^{10}$ per $\mathrm{mm}^{2}$. The increase is 85 and $50 \%$ for $1.23 \mathrm{~V}$ and $1.9 \mathrm{~V} v$ s. RHE, respectively.

An average density of $3.5 \times 10^{10}$ clusters per $\mathrm{mm}^{2}$ provides a footprint which would be approximately equivalent to 0.4 monolayers of $\mathrm{Ti}_{2000 \pm 54}$ clusters on an atomically flat surface, using diameters from the spherical cluster approximation. This density represents the deposition of only $2.8 \times 10^{-7} \mathrm{~g}$ of metal on the surface. A decrease in the photocurrent observed for the highest average density used, $7.5 \times 10^{10}$ clusters per $\mathrm{mm}^{2}$, could be attributed to the blocking of the $\mathrm{BiVO}_{4}$ surface to the water splitting reactions, leading to a dramatic increase in recombination.

Attempts were made to measure the Absorbed Photon Conversion Efficiency (APCE) of $\mathrm{BiVO}_{4}$ photoelectrodes before and after cluster deposition, in an effort to gain greater understanding of the effect mass-selected clusters had on the photoelectrochemical behavior. Although $\mathrm{BiVO}_{4}$ is a promising photoanodic material, it also known to have less than ideal photostability. ${ }^{51}$ Unfortunately it was found that during the long measurement times, required for accurate photocurrent measurements (required to calculate APCE), caused degradation of our $\mathrm{BiVO}_{4}$ electrodes. Stability measurements for $\mathrm{BiVO}_{4}$ electrodes were conducted under blue and white light illumination to evaluate their stability and it was confirmed the weak stability of $\mathrm{BiVO}_{4}$ electrodes (ESI Fig. S7 and S8†). 

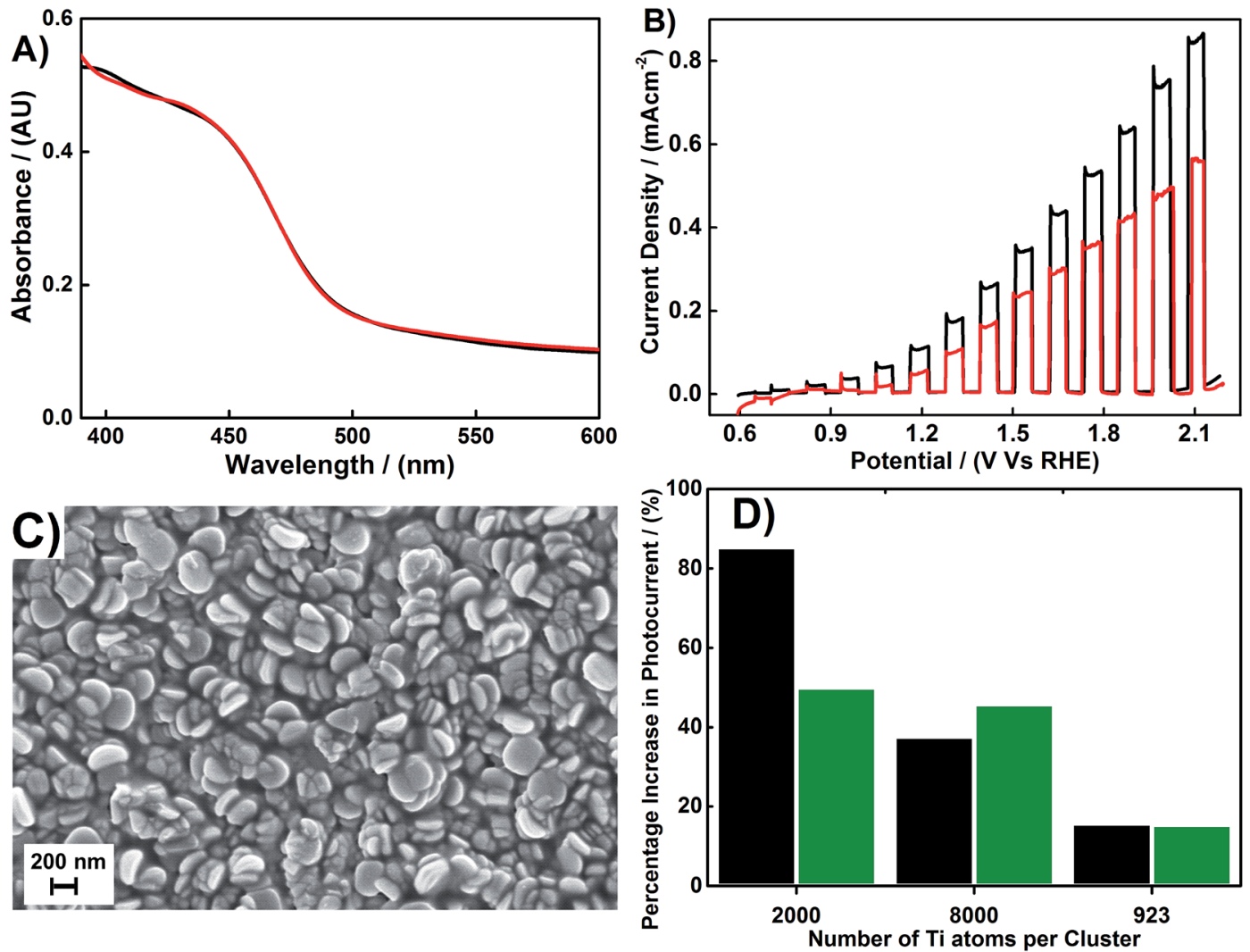

Fig. 5 (A) UV-visible absorption spectra for $\mathrm{BiVO}_{4}$ photoelectrodes with (black) and without (red) Ti $2000 \pm 54$ clusters; (B) chopped J-V curves showing $\mathrm{BiVO}_{4}$ with (black) and without (red) $\mathrm{Ti}_{2000 \pm 54}$ clusters; (C) SEM image of a typical BiVO ${ }_{4}$ morphology produced by AACVD at 50 000 $\times$ magnification; (D) percentage increase in photocurrent vs. cluster size at two different potentials, $1.23 \mathrm{~V}$ (black) and $1.9 \mathrm{~V}$ (green) vs. RHE.

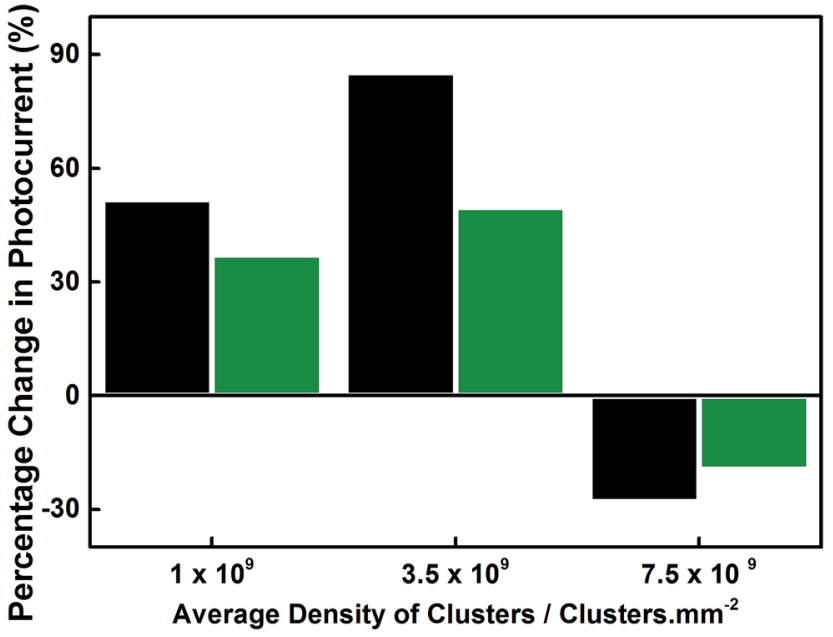

Fig. 6 Percentage photocurrent change resulting from $\mathrm{Ti}_{2000 \pm 54}$ cluster deposition on $\mathrm{BiVO}_{4}$ as a function of average density. Photocurrent analyzed at $1.23 \mathrm{~V}$ (black) and $1.9 \mathrm{~V}$ (green) vs. RHE.

Control experiments were also conducted to investigate the photoelectrochemical properties of mass-selected Ti-nanoclusters themselves, whereby $\mathrm{Ti}_{2000 \pm 54}$ mass-selected clusters were deposited on bare fluorine-doped tin oxide conducting glass substrates (ESI Fig. S9†) at a density of approximately
$3.5 \times 10^{10}$ clusters per $\mathrm{mm}^{2}$. These control samples showed no photoresponse under identical 3-electrode measurement conditions to that of $\mathrm{BiVO}_{4}$ samples. This suggests that the $\mathrm{TiO}_{2}$ nanoclusters used in this study are neither photoactive per se, nor acting as photosensitizers for $\mathrm{BiVO}_{4}$, which agrees well with the literature. ${ }^{52}$ Furthermore, the observed photocurrent enhancement for cluster modified $\mathrm{BiVO}_{4}$ electrodes is very unlikely due to optical effects, as the size range of $\mathrm{TiO}_{2}$ nanoclusters used in this study would show quantum confinement effects, increasing the width of the band gap, thereby shifting the optical absorption edge further towards the UV region of the solar spectrum where the photon density is very low. ${ }^{53}$

In an effort to further understand the mechanism for the photocurrent increase, photoelectrochemical impedance spectroscopy was carried out on the $\mathrm{BiVO}_{4}$ photoelectrodes modified with oxidized mass-selected Ti clusters. Fig. 7 shows the Nyquist plots for $\mathrm{BiVO}_{4}$ photoelectrodes before and after deposition of $\mathrm{Ti}_{2000 \pm 54}$ clusters deposited at $3.5 \times 10^{10}$ clusters per $\mathrm{mm}^{2}$ average density. The data was fitted with an equivalent circuit, with circuit components for the solution resistance, $R_{\mathrm{s}}$, the charge transfer resistance, $R_{\mathrm{ct}}$ and a constant phase element for the semiconductor-electrolyte interface, as shown in Fig. 7 inset. An arc is seen in the Nyquist plot, which corresponds to the charge transfer resistance between the electrode-electrolyte interface. $R_{\mathrm{ct}}$ is calculated by subtracting the low frequency $x$ axis intercept from the high frequency $x$-axis intercept. ${ }^{51}$ Thus 


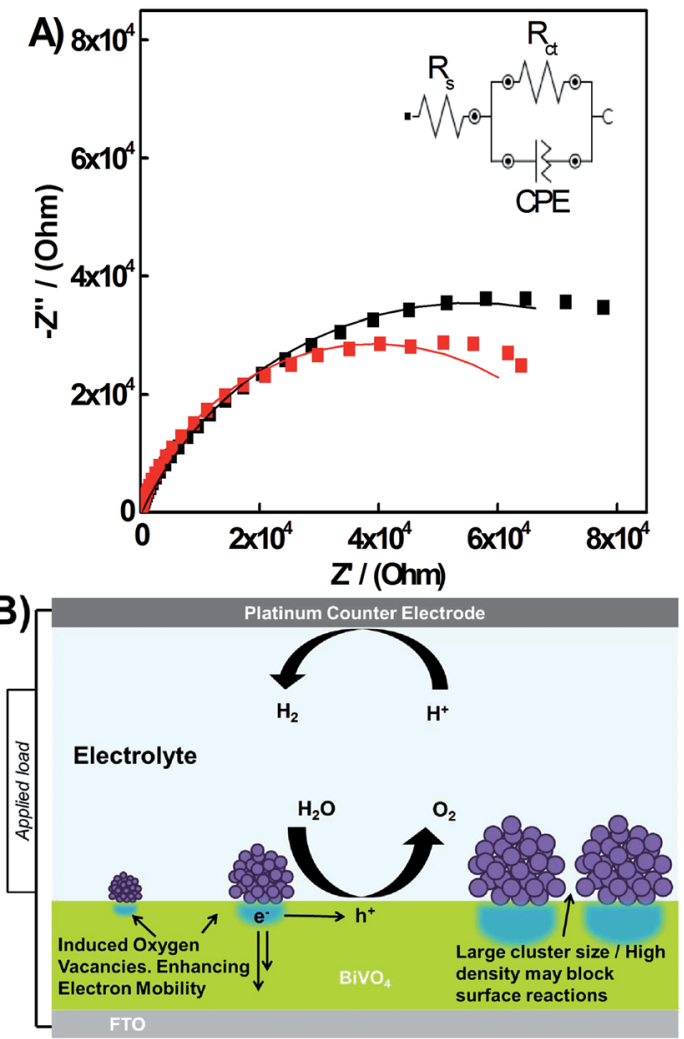

Fig. 7 (A) Nyquist plot for $\mathrm{BiVO}_{4}$ photoelectrodes measured, under blue light illumination at $1.23 \mathrm{~V}$ vs. RHE, with (red) and without (black) $\mathrm{Ti}_{2000 \pm 54}$ clusters deposited at an average density of $3.5 \times 10^{10}$ clusters per $\mathrm{mm}^{2}$; (B) schematic illustration outlining the photocurrent enhancement mechanism of different sized clusters on the surface of $\mathrm{BiVO}_{4}$.

$R_{\mathrm{ct}}$ can be calculated as $110 \mathrm{k} \Omega$ and $78 \mathrm{k} \Omega$ for before and after cluster deposition respectively. This clear decrease in the charge transfer resistance, of over $30 \mathrm{k} \Omega$, indicates that the number of holes reaching the electrolyte has increased. It is possible that the decrease in charge transfer resistance is caused by an improvement in the electron mobility. ${ }^{54}$ Further understanding of the reaction kinetics on our electrodes via PEIS and phenomenological models would be ideal, ${ }^{55}$ but is hindered by the nanostructured morphology of these electrodes, which leads to non-ideal behavior of the space charge layer and the poor photostability of the semiconductor material for lengthy measurements.

Determining the precise mechanism for the photocurrent enhancement on $\mathrm{TiO}_{2}$ nanocluster modified $\mathrm{BiVO}_{4}$ photoelectrodes has been challenging due to complications arising from stability issues; PEIS studies of $\mathrm{BiVO}_{4}$ require samples to be illuminated for a prolonged period of time. One potential route for photocurrent enhancement of the $\mathrm{BiVO}_{4}$ electrodes, that has been suggested in previous studies, is an increase in the band bending at the $\mathrm{BiVO}_{4}$-electrolyte interface. ${ }^{56,57}$ Increasing the band bending at the $\mathrm{BiVO}_{4}$-electrolyte interface will enhance the electron-hole separation, thus reducing photogenerated charge carrier recombination, increasing the photocurrent. ${ }^{58}$ Band bending from small Schottky contacts on a semiconductor can extend laterally a great distance from the contact itself, therefore it is reasonable to assume that the clusters have a wide reaching effect, possibly to a depth ten times the contact diameter, over the surface of the $\mathrm{BiVO}_{4}$, even with their small size. ${ }^{59}$

Another potential explanation for the photocurrent increase could be that mass-selected cluster deposition introduces more oxygen vacancies into the $\mathrm{BiVO}_{4}$ thin film, primarily at the surface. It is well known that oxygen vacancies in $\mathrm{BiVO}_{4}$ lead to the introduction of mobile electrons, enhancing electron transport, thus reducing bulk recombination. ${ }^{60,61}$ In $\mathrm{BiVO}_{4}$ the majority charge carrier transport is much slower than the minority carrier transport. In the high vacuum environment of the cluster source, when bare Ti clusters land on $\mathrm{BiVO}_{4}$, they would become partially oxidized by reacting with oxygen from the surface of the $\mathrm{BiVO}_{4}$ lattice, partially reducing the $\mathrm{BiVO}_{4}$ surface. This is supported by the Ti-O bond dissociation energy being greater than the $\mathrm{V}-\mathrm{O}$ bond dissociation energy, $666.5 \mathrm{~kJ}$ $\mathrm{mol}^{-1}$ and $637 \mathrm{~kJ} \mathrm{~mol}^{-1}$ respectively. ${ }^{62}$ In this scenario, the number of oxygen vacancies introduced into the $\mathrm{BiVO}_{4}$ will depend on the surface area of the semiconductor that is in contact with the Ti cluster during deposition. Small clusters, such as $\mathrm{Ti}_{923 \pm 25}$, would introduce fewer oxygen vacancies compared to larger clusters, such as the $\mathrm{Ti}_{8000 \pm 216}$, when deposited at the same density, as less Ti from the clusters would be in contact with the $\mathrm{BiVO}_{4}$. It is possible, however, that the larger clusters also screen parts of the surface, preventing the available photogenerated charge to undergo the water oxidation reaction. This may explain why the medium sized cluster is seen to provide the highest photocurrent enhancement, as two competing effects, the introduction of oxygen vacancies and the screening of the surface, both occur simultaneously. If this mechanism is correct, then the size dependency is essentially an extension of the coverage effect, which is also shown in this work. In this case fewer clusters, $1 \times 10^{10}$ clusters per $\mathrm{mm}^{2}$, would lead to fewer oxygen vacancies compared to a greater numbers of clusters, $7.5 \times 10^{10}$ clusters per $\mathrm{mm}^{2}$. Again, critically, too many clusters present on the surface would screen it to the water oxidation reaction, which in the case of $7.5 \times 10^{10}$ clusters per $\mathrm{mm}^{2}$, actually leads to a decrease in the photocurrent. We believe that even though the interaction is potentially determined through the degree of surface contact with the Ti clusters during deposition, the most accurate way of manipulating this mechanism is through control of the size of the nanoclusters, achieved with the cluster source mass filter, rather than relying on the density of clusters. Small changes in the cluster mass will allow incremental changes to the surface coverages, more accurately, than using the average cluster density. Thus, our observation is that the cluster size dependent affect appeared to be dominant.

\section{Conclusions}

In summary, this work demonstrates that the deposition of a remarkably small quantity of mass-selected clusters on the surface of a semiconductor substrate can lead to a significant change in its photoelectrochemical properties. A size dependency was found to exist for the photocurrent enhancement and 
ultimately an $85 \%$ improvement was seen in the photocurrent of $\mathrm{BiVO}_{4}$ with $\mathrm{Ti}_{2000 \pm 54}$ deposited at approximately 0.4 monolayers. We have hypothesized that the size of the clusters and the density of deposition may increase the number of oxygen vacancies within the surface layers of the $\mathrm{BiVO}_{4}$ thin film, leading to enhanced electron transport which may reduce recombination. These results highlight the benefit of incorporating a sub monolayer quantity of nanoclusters on semiconductor surfaces in order to enhance the interfacial charge transfer properties, emphasizing the significant potential of this new surface modification method. In general, our method of modification of semiconductor surfaces will open further opportunities in development of metal oxide semiconductor interface based applications such as PEC devices and sensors.

\section{Author contributions}

The manuscript was written through contributions of all authors. All authors have given approval to the final version of the manuscript. All authors contributed to writing the manuscript. AM carried out cluster deposition, sample characterization and PEC measurements and analysis of results. SRP assisted with cluster deposition and interpretation of the results (including STEM). IMO performed STEM characterization and analyzed the results. KGUW and REP conceived and supervised the project.

\section{Funding sources}

This research was funded by the Engineering and Physical Research Council (Grant no. EP/G037116/1) through a PhD studentship with the Doctoral Training Centre for Hydrogen, Fuel Cells and their Applications.

\section{Acknowledgements}

A McInnes acknowledges the support the Doctoral Training Centre for Hydrogen, Fuel Cells and their Applications. The work was supported by colleagues at the Energy Research Laboratory, Department of Chemistry, Loughborough University and the Nanoscale Physics Research Laboratory, School of Physics and Astronomy, University of Birmingham.

\section{References}

1 M. S. Prévot and K. Sivula, J. Phys. Chem. C, 2013, 117, 1787917893.

2 A. Fujishima and K. Honda, Nature, 1972, 238, 37-38.

3 A. A. Tahir, T. A. N. Peiris and K. G. U. Wijayantha, Chem. Vap. Deposition, 2012, 18, 107-111.

4 M. G. Walter, E. L. Warren, J. R. McKone, S. W. Boettcher, Q. Mi, E. A. Santori and N. S. Lewis, Chem. Rev., 2010, 110, 6446-6473.

5 R. Daghrir, P. Drogui and D. Robert, Ind. Eng. Chem. Res., 2013, 52, 130226090752004.

6 F. Gracia, J. P. Holgado and R. Gonza, Langmuir, 2004, 20, 1688-1697.
7 H. Tang, K. Prasad, R. Sanjines, P. E. Schmid and F. Levy, J. Appl. Phys., 1994, 75, 2042-2047.

8 F. Cao, G. Oskam, P. C. Searson, J. M. Stipkala, T. A Heimer, F. Farzad and G. J. Meyer, J. Phys. Chem., 1995, 99, 1197411980.

9 B. Schwenzer, L. Wang, J. S. Swensen, A. B. Padmaperuma, G. Silverman, R. Korotkov and D. J. Gaspar, Langmuir, 2012, 28, 10072-10081.

10 M. Ni, M. K. H. Leung, D. Y. C. Leung and K. Sumathy, Renewable Sustainable Energy Rev., 2007, 11, 401-425.

11 A. Wolcott, W. A. Smith, T. R. Kuykendall, Y. Zhao and J. Z. Zhang, Small, 2009, 5, 104-111.

12 I. S. Cho, Z. Chen, A. J. Forman, D. R. Kim, P. M. Rao, T. F. Jaramillo and X. Zheng, Nano Lett., 2011, 11, 4978-4984.

13 Q. Zhang, W. Gong, J. Wang, X. Ning, Z. Wang, X. Zhao, W. Ren and Z. Zhang, J. Phys. Chem. C, 2011, 115, 2524125246.

14 J. Chen, Q. Zhang, Y. Wang and H. Wan, Adv. Synth. Catal., 2008, 350, 453-464.

15 F. J. Perez-Alonso, D. N. McCarthy, A. Nierhoff, P. Hernandez-Fernandez, C. Strebel, I. E. L. Stephens, J. H. Nielsen and I. Chorkendorff, Angew. Chem., Int. Ed., 2012, 51, 4641-4643.

16 L. M. Peter and K. G. Upul Wijayantha, ChemPhysChem, 2014, 15, 1983-1995.

17 S. Y. Chae, M. K. Park, S. K. Lee, T. Y. Kim, S. K. Kim and W. I. Lee, Chem. Mater., 2003, 15, 3326-3331.

18 M. Pal, J. GarciaSerrano, P. Santiago and U. Pal, J. Phys. Chem. C, 2007, 111, 96-102.

19 R. E. Palmer, S. Pratontep and H.-G. Boyen, Nat. Mater., 2003, 2, 443-448.

20 C. Binns, Surf. Sci. Rep., 2001, 44, 1-49.

21 J. P. Wilcoxon, J. E. Martin and P. Provencio, J. Chem. Phys., 2001, 115, 998.

22 W. Marine, L. Patrone, B. Luk'Yanchuk and M. Sentis, Appl. Surf. Sci., 2000, 154, 345-352.

23 M. Imanaka, T. Katayama, Y. Ohshiro, S. I. Watanabe, H. Arai and T. Nakagawa, Rev. Sci. Instrum., 2004, 75, 1907-1909.

24 S. G. Hall, M. B. Nielsen, A. W. Robinson and R. E. Palmer, Rev. Sci. Instrum., 1997, 68, 3335-3339.

25 S. Pratontep, S. J. Carroll, C. Xirouchaki, M. Streun and R. E. Palmer, Rev. Sci. Instrum., 2005, 76, 45103.

26 K. Wegner, P. Piseri, H. V. Tafreshi and P. Milani, J. Phys. D: Appl. Phys., 2006, 39, R439-R459.

27 A. Majumdar, D. Köpp, M. Ganeva, D. Datta, S. Bhattacharyya and R. Hippler, Rev. Sci. Instrum., 2009, 80, 095103.

28 T. G. Dietz, M. A. Duncan, D. E. Powers and R. E. Smalley, J. Chem. Phys., 1981, 74, 6511-6512.

29 B. von Issendorff and R. E. Palmer, Rev. Sci. Instrum., 1999, 70, 4497-4501.

30 S. Srivastava, J. P. Thomas, M. A. Rahman, M. Abd-Ellah, M. Mohapatra, D. Pradhan, N. F. Heinig and K. T. Leung, ACS Nano, 2014, 8, 11891-11898.

31 J. R. McKone, N. S. Lewis and H. B. Gray, Chem. Mater., 2014, 26, 407-414. 
32 P. Brack, J. S. Sagu, T. A. N. Peiris, A. McInnes, M. Senili, K. G. U. Wijayantha, F. Marken and E. Selli, Chem. Vap. Deposition, 2015, 21, 41-45.

33 F. F. Abdi, L. Han, A. H. M. Smets, M. Zeman, B. Dam and R. van de Krol, Nat. Commun., 2013, 4, 2195.

34 Y. Pihosh, I. Turkevych, K. Mawatari, J. Uemura, Y. Kazoe, S. Kosar, K. Makita, T. Sugaya, T. Matsui, D. Fujita, M. Tosa, M. Kondo and T. Kitamori, Sci. Rep., 2015, 5, 11141.

35 X. Shi, I. Y. Choi, K. Zhang, J. Kwon, D. Y. Kim, J. K. Lee, S. H. Oh, J. K. Kim and J. H. Park, Nat. Commun., 2014, 5, 4775.

36 H. S. Park, H. C. Lee, K. C. Leonard, G. Liu and A. J. Bard, ChemPhysChem, 2013, 14, 2277-2287.

37 L. H. Mascaro, A. Pockett, J. M. Mitchels, L. M. Peter, P. J. Cameron, V. Celorrio, D. J. Fermin, J. S. Sagu, K. G. U. Wijayantha, G. Kociok-Köhn and F. Marken, J. Solid State Electrochem., 2014, 31-35.

38 A. McInnes, J. S. Sagu and K. G. U. Wijayantha, Mater. Lett., 2014, 137, 214-217.

39 A. A. Tahir, M. A. Mat-Teridi and K. G. U. Wijayantha, Phys. Status Solidi RRL, 2014, 8, 976-981.

40 R. L. Johnston, Atomic and Molecular Clusters, Taylor and Francis, London, 2002.

41 F. Yin, Z. W. Wang and R. E. Palmer, J. Am. Chem. Soc., 2011, 133, 10325-10327.

42 C. E. Blackmore, N. V. Rees and R. E. Palmer, Phys. Chem. Chem. Phys., 2015, 17, 28005-28009.

43 N. Jian and R. E. Palmer, J. Phys. Chem. C, 2015, 119, 1111411119.

44 D. Pearmain, S. J. Park, A. Abdela, R. E. Palmer and Z. Y. Li, Nanoscale, 2015, 7, 19647-19652.

45 K. J. Hu, S. R. Plant, P. R. Ellis, C. M. Brown, P. T. Bishop and R. E. Palmer, J. Am. Chem. Soc., 2015, 137, 15161-15168.
46 M. Drabik, A. Choukourov, A. Artemenko, J. Matousek, O. Polonskyi, P. Solar, J. Pesicka, J. Lorincik, D. Slavinska and H. Biederman, Surf. Coat. Technol., 2011, 205, 48-52.

47 J. Sun, X. Li, Q. Zhao, J. Ke and D. Zhang, J. Phys. Chem. C, 2014, 118, 10113-10121.

48 L. H. Dall'Antonia, N. R. de Tacconi, W. Chanmanee, H. Timmaji, N. Myung and K. Rajeshwar, Electrochem. Solid-State Lett., 2010, 13, D29.

49 T. W. Kim and K.-S. Choi, Science, 2014, 343, 990-994.

50 L. M. Peter, K. G. U. Wijayantha and A. A. Tahir, Faraday Discuss., 2012, 155, 309.

51 C. Ding, J. Shi, D. Wang, Z. Wang, N. Wang, G. Liu, F. Xiong and C. Li, Phys. Chem. Chem. Phys., 2013, 15, 4589.

52 K. Fujihara, S. Izumi, T. Ohno and M. Matsumura, J. Photochem. Photobiol., A, 2000, 132, 99-104.

53 K. M. Reddy, S. V. Manorama and A. R. Reddy, Mater. Chem. Phys., 2003, 78, 239-245.

54 M. X. Tan, P. E. Laibinis, S. T. Nguyen, J. M. Kesselman, C. E. Stanton and N. S. Lewis, in Progress in Inorganic Chemistry, 1994, pp. 21-144.

55 K. G. G. Upul Wijayantha, S. Saremi-Yarahmadi and L. M. Peter, Phys. Chem. Chem. Phys., 2011, 13, 5264-5270.

56 D. Eisenberg, H. S. Ahn and A. J. Bard, J. Am. Chem. Soc., 2014, 10-13.

57 S. Ho-Kimura, S. J. A. Moniz, A. D. Handoko and J. Tang, J. Mater. Chem. A, 2014, 2, 3948.

58 Z. Zhang and J. T. Yates, Chem. Rev., 2012, 112, 5520-5551.

59 C. Donolato, J. Appl. Phys., 2004, 95, 2184-2186.

60 Y. Liang, T. Tsubota, L. P. A. Mooij and R. van de Krol, J. Phys. Chem. C, 2011, 115, 17594-17598.

61 M. Li, L. Zhao and L. Guo, Int. J. Hydrogen Energy, 2010, 35, 7127-7133.

62 W. M. Haynes, Handbook of Chemistry and Physics, Taylor and Francis, 92nd edn, 2012. 